\title{
Modeling Partially Reliable Information Sources: a General Approach based on Dempster-Shafer Theory
}

\author{
Rolf Haenni \\ Institute of Computer Science and Applied Mathematics, University of Berne \\ CH-3012 Berne, Switzerland \\ Stephan Hartmann \\ Department of Philosophy, London School of Economics \\ London, WC2A 2AE, United Kingdom \\ Center for Junior Research Fellows, University of Konstanz \\ D-78457 Konstanz, Germany
}

\begin{abstract}
Combining testimonial reports from independent and partially reliable information sources is an important problem of uncertain reasoning. Within the framework of Dempster-Shafer theory, we propose a general model of partially reliable sources which includes several previously known results as special cases. The paper reproduces these results, gives a number of new insights, and thereby contributes to a better understanding of this important application of reasoning with uncertain and incomplete information. ${ }^{1,2}$
\end{abstract}

Key words: Dempster-Shafer Theory, Probabilistic Argumentation, Combining Testimonial Reports, Unreliable Sources, Sensor Fusion, Judgement Aggregation, Probabilistic Algorithms.

\footnotetext{
1 Research supported by (1) Swiss National Science Foundation, Project No. PP002-102652/1 (2) Alexander von Humboldt Foundation, (3) German Federal Ministry of Education and Research, (4) German Program for the Investment in the Future.

2 This is an extended version of a conference paper presented at the 7th International Conference on Information Fusion in Stockholm [1].

Email addresses: haenni@iam.unibe.ch (Rolf Haenni), S.Hartmann@lse.ac.uk (Stephan Hartmann).

$U R L$ : haenni.shorturl.com (Rolf Haenni).
} 


\section{Introduction}

An important application of uncertain reasoning is the problem of combining reports or data from partially reliable witnesses, experts, sensors, or measurement instruments. In legal cases, for example, testimonies from different witnesses must be taken into account. Due to the possible unreliability of witnesses, testimonies do typically not fully confirm each other. Very often, testimonies are even totally conflicting. Judges or jurors must then resolve such conflicts and pass their sentence accordingly. Similarly, if several reports from sensors or data obtained from different measurement instruments are given, it is typical to have conflicts or inconsistencies. This is again due to the possible unreliability of sensors or measurement instruments.

The common feature of situations like the ones mentioned above is that confirming and/or conflicting information is obtained from partially reliable information sources. The question of how to combine such information is a very general problem of uncertain reasoning. We will refer to it as the problem of partially reliable information sources.

There are numerous ways to attack this problem. In order to simplify matters, it is usually assumed that:

(1) There are only two alternative hypotheses Hyp and $\neg H y p$ (e.g. suspect $X$ is guilty vs. suspect $X$ is innocent).

(2) All sources are structurally identical and are therefore represented by one particular model and a corresponding set of characteristic parameters.

(3) The sources are conditionally independent given the hypothesis (the hypothesis is the only common parameter that determines the outcomes of their reports).

These assumptions may appear to be overly idealized, but one can imagine practical situations in which they are approximately correct (some examples are given in the following subsection). We will accept these three assumptions throughout this paper and postpone more general models to future publications. Because only two alternative hypotheses Hyp and $\neg H y p$ are considered, we can distinguish between positive reports and negative reports. In general, we consider $n$ positive and $m$ negative reports. $N=n+m$ is the total number of reports.

Any concrete model of partially reliable information sources is supposed to represent what is known about the source. This is an important remark, and it means that we distinguish strictly between the true behavior of the sources and our knowledge about it. Since the true behavior is often inaccessible or even unknowable, we must think of a model as an imprecise or incomplete 
description of the real world mechanisms that determine the comportment of an information source. An extreme (but valid) model is the case in which absolutely no information is available.

\subsection{Existing Models}

As we will see in Section 3, there is huge number of possible models of partially reliable information sources. A few of them are well-known in the literature and widely applied in various domains. In the following, we give a non-comprehensive overview of such existing models. The first two models can be represented by respective Bayesian networks and require thus the existence of some prior information on the hypothesis. In the third and the fourth model, it is possible to include prior information on the hypothesis, but it is not mandatory. In all four models, the reports of the information sources are compiled into an overall probabilistic judgment. This paper will show how to derive these models within a more general framework.

\section{Approach No. 1}

A classical way to deal with partially reliable sources is to assume that there is a certain chance $p_{i}=\operatorname{Pr}\left(\operatorname{Re}_{i} \mid H y p\right)$ that source $i$ yields a positive report $\operatorname{Re} p_{i}$ if the hypothesis Hyp is true. On the other hand, there is certain probability $q_{i}=\operatorname{Pr}\left(\operatorname{Rep}_{i} \mid \neg H y p\right)$ that the source yields a positive report $R e p_{i}$ if Hyp is false. $q_{i}$ represents the so-called ratio of false positives and $\bar{p}_{i}=1-p_{i}$ the ratio of false negatives. These values are, for example, well documented for many medical tests.

If $N$ independent information sources of this type provide reports, we obtain the simple Bayesian network depicted in Fig. 1. The root node $H Y P$ is the parent node of $N$ report nodes $R E P_{1}$ to $R E P_{N}$. The possible values of $H Y P$ are $H y p$ and $\neg H y p$, and the possible values of $R E P_{i}$ are $R e p_{i}$ and $\neg R e p_{i}$.

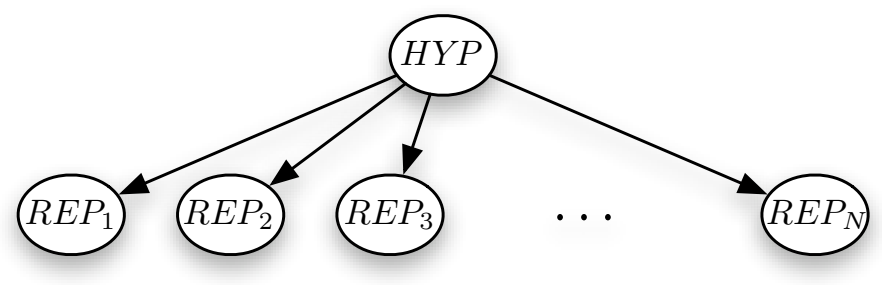

Fig. 1. A simple Bayesian model of partially reliable information sources.

Let $h=\operatorname{Pr}($ Hyp $)$ and $\bar{h}=1-h$ denote the prior probabilities of Hyp and $\neg H y p$, respectively. If $n$ reports are positive and the remaining $m=N-n$ 
reports are negative, and if we assume to have equal parameters $p_{i}=p$ and $q_{i}=q$ for all $1 \leq i \leq N$, then we can apply Bayes' theorem to obtain the following posterior probability for Hyp:

$$
\begin{aligned}
\operatorname{Pr}^{\prime}(\text { Hyp }) & =\operatorname{Pr}\left(H y p \mid \operatorname{Rep}_{1}, \ldots, \operatorname{Rep}_{n}, \neg \operatorname{Re} p_{n+1}, \ldots, \neg \operatorname{Re} p_{N}\right) \\
& =\frac{h}{h+\bar{h}(q / p)^{n}(\bar{q} / \bar{p})^{m}}
\end{aligned}
$$

For a detailed discussion and interpretation of this formula we refer to [2]. In Subsection 4.3 we will reproduce the same result as a special case of a more general model.

\section{Approach No. 2}

An alternative approach is to model partially reliable sources on the basis of reliability variables $R E L_{i}$ with $\operatorname{Rel}_{i}$ and $\neg R e l_{i}$ as possible values (see [2] for a detailed discussion). It is then assumed that the report of a reliable source (represented by $R e l_{i}$ ) is positive whenever the hypothesis is true and negative whenever the hypothesis is false.

More formally, we have $\operatorname{Pr}\left(\operatorname{Rep}_{i} \mid H y p, \operatorname{Re}_{i}\right)=1$ and $\operatorname{Pr}\left(\operatorname{Rep}_{i} \mid \neg H y p, \operatorname{Re}_{i}\right)=0$. On the other hand, if source $i$ is unreliable, then we suppose the outcome of $R E P_{i}$ to depend only on a randomization parameter $r_{i}$ no matter what the true state of $H Y P$ is. In other words, we assume $\operatorname{Pr}\left(\operatorname{Rep}_{i} \mid H y p, \neg \operatorname{Re}_{i}\right)=$ $\operatorname{Pr}\left(\operatorname{Rep}_{i} \mid \neg H y p, \neg \operatorname{Re}_{i}\right)=r_{i}$. Such a constellation leads to the Bayesian network depicted in Fig. 2, in which $H Y P$ and $R E L_{i}$ are the parents of $R E P_{i}$ for all $1 \leq i \leq N$.

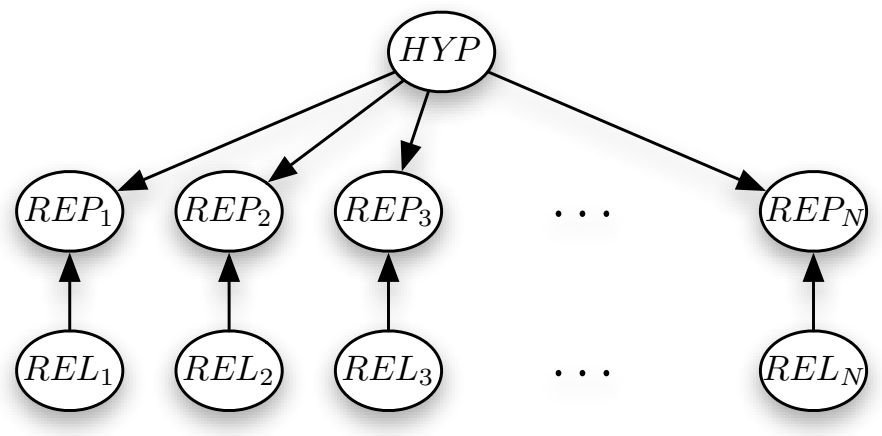

Fig. 2. Another simple Bayesian model of partially reliable information sources.

Consider again $n$ positive and $m$ negative reports. If we assume the same prior probability $\operatorname{Pr}\left(\operatorname{Re}_{i}\right)=\rho$ and the same randomization parameter $r_{i}=r$ for 
all $1 \leq i \leq N$, we can apply Bayes' theorem to obtain the following posterior probability for Hyp:

$$
\begin{aligned}
\operatorname{Pr}^{\prime}(\text { Hyp }) & =\operatorname{Pr}\left(\operatorname{Hyp} \mid \operatorname{Rep}_{1}, \ldots, \operatorname{Rep}_{n}, \neg \operatorname{Re} p_{n+1}, \ldots, \neg \operatorname{Re} p_{N}\right) \\
& =\frac{h}{h+\bar{h}\left(\frac{\bar{\rho} r}{1-\bar{\rho} \bar{r}}\right)^{n}\left(\frac{1-\overline{\bar{\rho}} r}{\bar{\rho} \bar{r}}\right)^{m}}
\end{aligned}
$$

Again, we refer to [2] for a detailed discussion of this result and its relationship to (1). We will also reproduce this formula in Subsection 4.4 as a special case of the general model.

Approach No. 3

A third simple model of partially reliable sources results from looking at source $i$ as somebody who is either telling the truth or who is purposely lying due to some lack of veracity. Let $r$ and $\bar{r}=1-r$ be the respective probabilities. Furthermore, in order to simplify matters, suppose that all $N$ information sources provide positive reports. This means that either they are all telling the truth with probability $r^{N}$ or they are all lying with probability $\bar{r}^{N}$, i.e. all other configurations are logically impossible. Obviously, Hyp must be true in the first and false in the second case. This leads to a formula for $\operatorname{Pr}(\operatorname{Hyp})$ that goes back to Laplace (see Chapt. XI of [3]) and is closely related to the Condorcet Jury Theorem discussed in social choice theory (see, amongst many others, $[4-7])$ :

$$
\operatorname{Pr}(\text { Hyp })=\frac{r^{N}}{r^{N}+\bar{r}^{N}}=\frac{1}{1+(\bar{r} / r)^{N}}
$$

It is remarkable that (3) does not depend on a prior distribution of $H Y P$. This raises the controversial question of whether this formula is a proper posterior probability in a Bayesian sense. Boole gives a similar formula that includes a prior distribution of $H Y P$ (see Chapt. XXI of [8]). He considers the isomorphic problem of $N$ independent jurors rendering an unanimous verdict. In Subsection 4.2, we will reproduce these formulas (both the one with and the one without a prior distribution over $H Y P$ ) in a general form for $n$ positive and $m$ negative reports. We will then get a clear interpretation of (3) and a better understanding of its relationship to a purely Bayesian analysis. 
Suppose that the source always (with absolute certainty) yields a positive report if Hyp is true, but if Hyp is false, it generates the report at random. Sources of this type are the basis of so-called probabilistic or randomized algorithms [9], among which probabilistic primality tests are the most famous instances. They do not determine with certainty whether a number is prime or not, but for an increasing running time, the error rate diminishes quickly towards zero. This is acceptable for many practical applications such as publickey cryptography.

The key mechanism of probabilistic primality tests (and probabilistic algorithms in general) is a repeated test that behaves like an information source of the above-mentioned type. This means that negative reports only occur (at random), if the hypothesis is false, i.e. they imply (with absolute certainty) that the number is not prime. On the other hand, nothing is proved if the report is positive, although every positive report increases the chance of having a prime number at hand. Suppose we get a sequence of $N$ positive reports, and let $r=\operatorname{Pr}\left(\operatorname{Rep}_{i} \mid \neg H y p\right)$ be the ratio of false negatives, then the probability of having a prime number is

$$
\operatorname{Pr}(H y p)=1-r^{N}
$$

which converges quickly towards 1 if $r$ is reasonably small. Again, no prior knowledge on $H Y P$ is needed. With a ratio of false negatives $r=\frac{1}{4}$, the best known probabilistic primality test is the so-called Miller-Rabin test [10].

\subsection{Goals and Overview}

The goal of this paper is to define a general model of partially reliable sources from which results like the ones mentioned above drop out as special cases. We choose Dempster-Shafer theory of evidence (DST) [11-13] and the closely related theory of probabilistic argumentation [14-17] as the underlying mathematical theories of uncertain reasoning. By looking at the problem of partially reliable information sources from the perspective of probabilistic argumentation, we will get a very clear picture of how the general model relates to more specific models such as the ones discussed above. We will also see that prior knowledge on $H Y P$, if available, is an important parameter. But interesting results are also obtained for the case where no prior knowledge is available. In fact, prior knowledge turns out to be a continuous quantity with "no prior knowledge" and $h=\operatorname{Pr}(H y p)$ at the opposite ends of the spectrum. 
Section 2 describes the general model and presents the main results. Section 3 proposes a map with numerous concrete models and links them with the main results of Section 2. Section 4 analyses the most interesting concrete models more deeply and reveals the relationship to and between the introductory models of Section 1. Section 5 adds some concluding remarks and raises some open questions.

\section{The General Model}

From an abstract point of view, we can say that every information source $i$ delivers some information $\mathcal{I}_{i}$ about the set of variables $d\left(\mathcal{I}_{i}\right)=\left\{H Y P, R E P_{i}\right\}$ called domain of $\mathcal{I}_{i}$. The only common variable over all $\mathcal{I}_{i}$ is $H Y P$, the one we are interested in. This reflects our assumption of conditionally independent sources given the hypothesis. As a consequence, before combining the various pieces of information $\mathcal{I}_{i}$, it is possible to focus or marginalize them to the common domain $\{H Y P\}$. In other words, every information source $i$ delivers some information $\mathcal{H}_{i}=\mathcal{I}_{i}^{\downarrow\{H Y P\}}$ about $H Y P$. Finally, if we suppose $\mathcal{H}_{0}$ to represent some given prior knowledge about $H Y P$, the problem is to compute the combined information

$$
\mathcal{H}=\mathcal{H}_{0} \otimes \mathcal{H}_{1} \otimes \cdots \otimes \mathcal{H}_{N}
$$

and to use $\mathcal{H}$ in order to draw conclusions about $H Y P$. This general analysis of the problem of partially reliable sources is in the spirit of Kohlas' theory of information algebras [18]. Its goal is to analyze algebraic systems obtained from applying two basic operations called combination $\otimes: \Psi \times \Psi \longrightarrow \Psi$ and marginalization $\downarrow: \Psi \times D \longrightarrow \Psi$ to a set of information pieces $\Psi$ and a set of domains $D$.

\subsection{The General Case}

From now on, suppose that every piece of information $\mathcal{H}_{i}$ is represented by an additive mass functions $m_{i}: 2^{H Y P} \longrightarrow[0,1]$ in the sense of DempsterShafer theory [11-13]. Note that in our further formal analysis, we will exclusively work with mass functions as representatives of the given knowledge. Because $H Y P$ is a binary variable, there are four possible subsets $\emptyset,\{H y p\}$, $\{\neg H y p\}$, and $\{H y p, \neg H y p\}$ to be considered. As a consequence, the corresponding masses $m_{i}(\emptyset), m_{i}(\{H y p\}), m_{i}(\{\neg H y p\})$, and $m_{i}(\{H y p, \neg H y p\})$ have to sum up to 1 . This means that one can fully specify every function $m_{i}$ by three parameters only, for example by 


$$
\begin{aligned}
x_{i} & :=m_{i}(\{H y p\})+m_{i}(\{H y p, \neg H y p\})=1-m_{i}(\{\neg H y p\})-m_{i}(\emptyset), \\
y_{i} & :=m_{i}(\{\neg H y p\})+m_{i}(\{H y p, \neg H y p\})=1-m_{i}(\{H y p\})-m_{i}(\emptyset) \\
z_{i} & :=m_{i}(\{H y p, \neg H y p\})
\end{aligned}
$$

This particular choice of three parameters seems arbitrary at first sight, but it will help us to get the most compact formulation of the main results of this paper (e.g. in Theorem 1). At this point, we do not further try to interpret the meaning of $x_{i}, y_{i}$, and $z_{i}$. We will see in Section 3 that such an interpretation depends crucially on the concrete model under consideration. The idea here is to generate general results in the framework of DST. The interpretation of the results will become clear in Section 4 when concrete models are considered.

If there are $N+1$ mass functions as specified above (one for the prior knowledge and $N$ for the reports), then $m=m_{0} \otimes \cdots \otimes m_{N}$ denotes the combined mass function obtained from applying Dempster's rule of combination $\otimes[12,13] .^{3}$ With respect to the combined mass function $m$, which represents the aggregated information from all sources, we are interested in the degree of support defined as

$$
d s p(\{H y p\})=\frac{m(\{H y p\})}{1-m(\emptyset)}
$$

and the degrees of possibility defined as

$$
\operatorname{dps}(\{H y p\})=1-d s p(\{\neg H y p\}) \cdot{ }^{4}
$$

This definition implies $d s p(\{H y p\}) \leq d p s(\{H y p\})$ for all possible hypothesis Hyp. Note that it is possible to interpret the margin $d p s(\{H y p\})-d s p(\{H y p\})$ as a quantitative measure of our ignorance with respect to Hyp [19].

3 Without loss of generality, we suppose $\otimes$ to denote the unnormalized version of Dempster's rule. This is useful to keep the proof of the main theorems as simple as possible. Furthermore, for a given set of reports, it may be useful to interpret $m(\emptyset)$ as a quantitative measure of the overall conflict between the sources.

4 In accordance with the theory of probabilistic argumentation [14-17], we prefer to speak about degree of support and degree of possibility of Hyp instead of belief $\operatorname{Bel}($ Hyp $)$ and plausibility $P l(H y p)$, respectively. In [17], degree of support is defined as the probability of provability of Hyp, which is an ordinary (additive) posterior probability of the event that the hypotheses Hyp is provable with respect to the given knowledge. One can also think of degrees of support as probabilistic weights of arguments and counter-arguments, respectively. 
Theorem 1 If $m_{0}$ to $m_{N}$ are mass functions over $H Y P$ as defined above, then the degree of support and the degree of possibility with respect to the combined mass function $m$ are determined by

$$
d s p(\{H y p\})=\frac{\prod_{i=0}^{N} x_{i}-\prod_{i=0}^{N} z_{i}}{\prod_{i=0}^{N} x_{i}+\prod_{i=0}^{N} y_{i}-\prod_{i=0}^{N} z_{i}}=1-\frac{\prod_{i=0}^{N} y_{i}}{\prod_{i=0}^{N} x_{i}+\prod_{i=0}^{N} y_{i}-\prod_{i=0}^{N} z_{i}},
$$

and

$$
\operatorname{dps}(\{H y p\})=\frac{\prod_{i=0}^{N} x_{i}}{\prod_{i=0}^{N} x_{i}+\prod_{i=0}^{N} y_{i}-\prod_{i=0}^{N} z_{i}},
$$

respectively.

Proof: We can use the fact that Dempster's rule of combination corresponds to multiplication of so-called commonality functions $q_{i}$ defined by $q_{i}(H)=$ $\sum\left\{m_{i}(A): A \supseteq H\right\}[12]$. In our concrete case, we have

$$
\begin{aligned}
& q_{i}(\emptyset)=m_{i}(\emptyset)+m_{i}(\{H y p\})+m_{i}(\{\neg H y p\})+m_{i}(\{H y p, \neg H y p\})=1, \\
& q_{i}(\{H y p\})=m_{i}(\{H y p\})+m_{i}(\{H y p, \neg H y p\})=x_{i}, \\
& q_{i}(\{\neg H y p\})=m_{i}(\{\neg H y p\})+m_{i}(\{H y p, \neg H y p\})=y_{i}, \\
& q_{i}(\{H y p, \neg H y p\})=m_{i}(\{H y p, \neg H y p\})=z_{i} .
\end{aligned}
$$

As a consequence, we get $q(\emptyset)=1, q(\{H y p\})=\prod_{i=0}^{N} x_{i}, q(\{\neg H y p\})=\prod_{i=0}^{N} y_{i}$, and $q(\{H y p, \neg H y p\})=\prod_{i=0}^{N} z_{i}$. This leads to $m(\emptyset)=1-\prod_{i=0}^{N} x_{i}-\prod_{i=0}^{N} y_{i}+$ $\prod_{i=0}^{N} z_{i}, m(\{H y p\})=\prod_{i=0}^{N} x_{i}-\prod_{i=0}^{N} z_{i}, m(\{\neg H y p\})=\prod_{i=0}^{N} y_{i}-\prod_{i=0}^{N} z_{i}$, and $m(\{H y p, \neg H y p\})=\prod_{i=0}^{N} z_{i}$. Finally, (8) results from applying (6) to $m(\{H y p\})$ and $m(\emptyset)$. Similarly, (9) is obtained from $(7)$ and $m(\{\neg H y p\})$.

For the particular case of two positive reports, the result of Theorem 1 corresponds to Lambert's discussion of the problem of unreliable testimonies (see Chapt. V of [20]).

\subsection{Identical Sources}

Now let there be $n$ positive and $m=N-n$ negative reports from structurally identical sources in the sense of the remark of Section 1. Suppose that the positive reports $m_{1}$ to $m_{n}$ are determined by parameters $x_{1}, y_{1}$, and $z_{1}$. Similarly, let $x_{2}, y_{2}$, and $z_{2}$ be the parameters for the negative reports $m_{n+1}$ to $m_{N}$. Finally, if $x, y$, and $z$ are the parameters of the prior knowledge $m_{0}$, we can transform the results of Theorem 1 into 


$$
\begin{aligned}
& d s p(\{H y p\})=\frac{x x_{1}^{n} x_{2}^{m}-z z_{1}^{n} z_{2}^{m}}{x x_{1}^{n} x_{2}^{m}+y y_{1}^{n} y_{2}^{m}-z z_{1}^{n} z_{2}^{m}}, \\
& d p s(\{H y p\})=\frac{x x_{1}^{n} x_{2}^{m}}{x x_{1}^{n} x_{2}^{m}+y y_{1}^{n} y_{2}^{m}-z z_{1}^{n} z_{2}^{m}} .
\end{aligned}
$$

These two formulas can be regarded as the key to a general solution of the problem of partially reliable sources in the restricted setting of independent but identical sources and two alternative hypotheses. At this point, we do not further comment on these results. Instead, we will introduce concrete models of partially reliable sources in Section 3. Various applications of the above formulas will be illustrated in Section 4 .

\subsection{Special Cases of Prior Knowledge}

Let us have a closer look at some special cases. First, consider the case where a prior distribution $h=\operatorname{Pr}($ Hyp $)$ is given. This means that $x=h, y=\bar{h}$, and $z=0$ are the parameters of the prior knowledge represented by $m_{0}$. Note that $z=0$ implies $d s p(\{H y p\})=d p s(\{H y p\})$. The expressions in (10) and (11) can then be transformed into

$$
d s p(\{H y p\})=\operatorname{dps}(\{H y p\})=\frac{h}{h+\bar{h}\left(y_{1} / x_{1}\right)^{n}\left(y_{2} / x_{2}\right)^{m}} .
$$

Furthermore, consider a case in which the prior knowledge is completely prejudiced by $h=0$ or $h=1$. Then the above formula simplifies to $d s p(\{H y p\})=$ $\operatorname{dps}(\{H y p\})=0$ and $\operatorname{dsp}(\{H y p\})=\operatorname{dps}(\{H y p\})=1$, respectively. Such a definite prejudice can therefore not be changed even if $|n-m|$ tends to $\infty$. Other interesting cases result from $x_{1}=y_{1}$ or $x_{2}=y_{2}$. It means that respective (positive or negative) reports do not make a difference and are thus of no value. Note how the corresponding expressions drop out of the denominator of (12). If all reports are valueless, that is if simultaneously $x_{1}=y_{1}$ and $x_{2}=y_{2}$, then $\operatorname{dsp}(\{H y p\})=\operatorname{dps}(\{H y p\})=h$, as one would expect.

Second, consider the case of $z=1$. This is a situation where no prior knowledge is available. It implies $x=1$ and $y=1$. As a consequence, we get

$$
\begin{aligned}
& d s p(\{H y p\})=\frac{x_{1}^{n} x_{2}^{m}-z_{1}^{n} z_{2}^{m}}{x_{1}^{n} x_{2}^{m}+y_{1}^{n} y_{2}^{m}-z_{1}^{n} z_{2}^{m}}, \\
& d p s(\{H y p\})=\frac{x_{1}^{n} x_{2}^{m}}{x_{1}^{n} x_{2}^{m}+y_{1}^{n} y_{2}^{m}-z_{1}^{n} z_{2}^{m}} .
\end{aligned}
$$


Note that the same result is obtained by omitting the prior knowledge $m_{0}$ from the beginning. In general, we have $0<z<1$. We will discuss in Subsection 3.6 how to interpret this general situation. Prior knowledge will then turn out to be a continuous quantity in which $z$ determines its strength and with "no prior knowledge" and $h=\operatorname{Pr}($ Hyp $)$ as opposite extreme cases.

\section{Taxonomy of Models}

So far, we have discussed the general case in which prior knowledge, positive reports, and negative reports are all characterized by three parameters. The question now is how to derive these parameters from concrete models like the ones introduced in Section 1.

Before doing so, we will first identify a variety of distinct models and discuss their respective relationships. To describe and classify these models, we use the expressive power of propositional logic as proposed by the theory of probabilistic argumentation $[15,16]$. This means that a specific model (M) will be expressed by a respective set $\Sigma_{(\mathrm{M})}$ of propositional sentences. The result will be a concise and comprehensive model taxonomy, which will serve as a general guideline for solving the problem of partially reliable information sources.

\subsection{Overview}

As mentioned before, it is important to distinguish between the true behavior of an information source and our knowledge about the source. Since the former is often inaccessible or unknowable, a model must always be seen as a representation of the latter. For example, there will be a model (I) in the model taxonomy that represents a situation of total ignorance about how the source generates its reports.

Without loss of generality, let's assume that a particular information source generates its report according to $s$ independent variables. One of them may be the hypothesis in question. If all $s$ variables are binary (exactly two possible outcomes), then there are $2^{s}$ possible configurations to be considered. In each of these $2^{s}$ cases, the source may either produce a positive or a negative report. Since we may or may not know how the source behaves for a specific configuration, we must distinguish between Rep, $\neg \operatorname{Rep}$, and $\operatorname{Rep} \vee \neg \operatorname{Rep}$ as possible outcomes. As a consequence, the total number of possible models is $m(s)=3^{2^{s}}$, that is $m(0)=3, m(1)=9, m(2)=81$, and so on. Note that every model obtained for $s-1$ variables is equivalent to a model obtained for $s$ variables. The model taxonomy will include models up to $s=4$. 
Among the variety of possible models, some are more interesting than others. Certain pairs of models, for example, are equivalent up to symmetry. We will try to restrict the model taxonomy to the most interesting ones, and exclude unnecessary redundancies. There will be a general distinction between complete and incomplete models. In total, we will discuss 21 different models of partially reliable information sources.

\subsection{Complete Models}

A model is called complete, if the way a report is generated is known for each possible configuration of relevant variables, otherwise it is called incomplete (or partial) [21]. We have thus $c(s)=2^{2^{s}}$ complete and $i(s)=m(s)-c(s)=$ $3^{2^{s}}-2^{2^{s}}$ incomplete models. Let us first try to classify the most interesting complete models. We will describe them both informally and with suitable sets $\Sigma_{(. .)}$of propositional sentences. The sets of propositions appearing in $\Sigma_{(\cdot .)}$ will be denoted by $V_{(\cdot .)}$, or $V$ for short, i.e. we have $s=|V|-1$. Our taxonomy of complete models will be exhaustive for $s \leq 2$ (up to symmetry).

Let us first look at the trivial case of $s=0$, which implies $V=\{\operatorname{Rep}\}$. The way the source generates its reports is thus not influenced by anything, not even by the hypothesis itself. In this sense, we speak of a non-informative source. For $s=0$, there are two different types of non-informative sources:

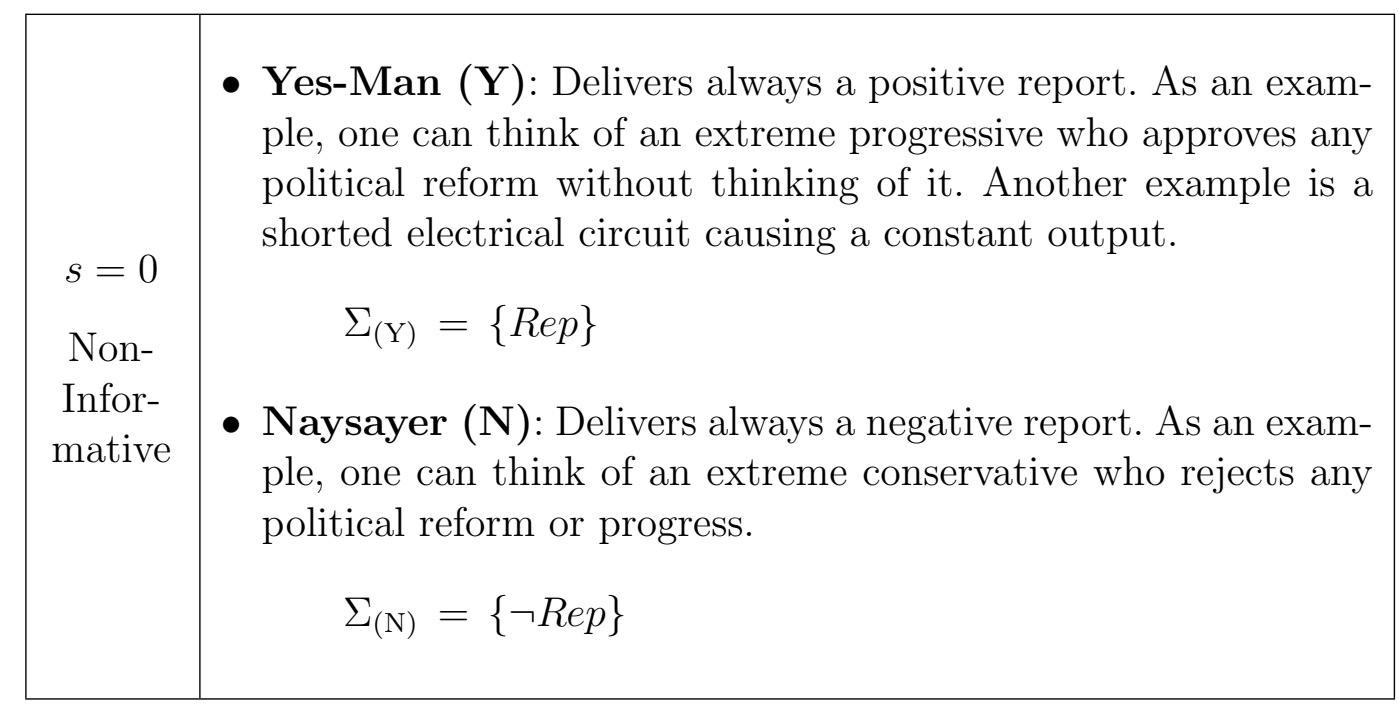

Now let's look at the possible models obtained for $s=1$. This means that the report depends on a single variable, and we must thus discriminate on whether this variable is the hypothesis or not. Formally, we will make a distinction between $V=\{H y p, R e p\}$ and $V=\{R, R e p\}$. In the former informative case, it is assumed that the source is conscious about the true state of the hypothesis (and makes use of it). There are two such alternatives: 


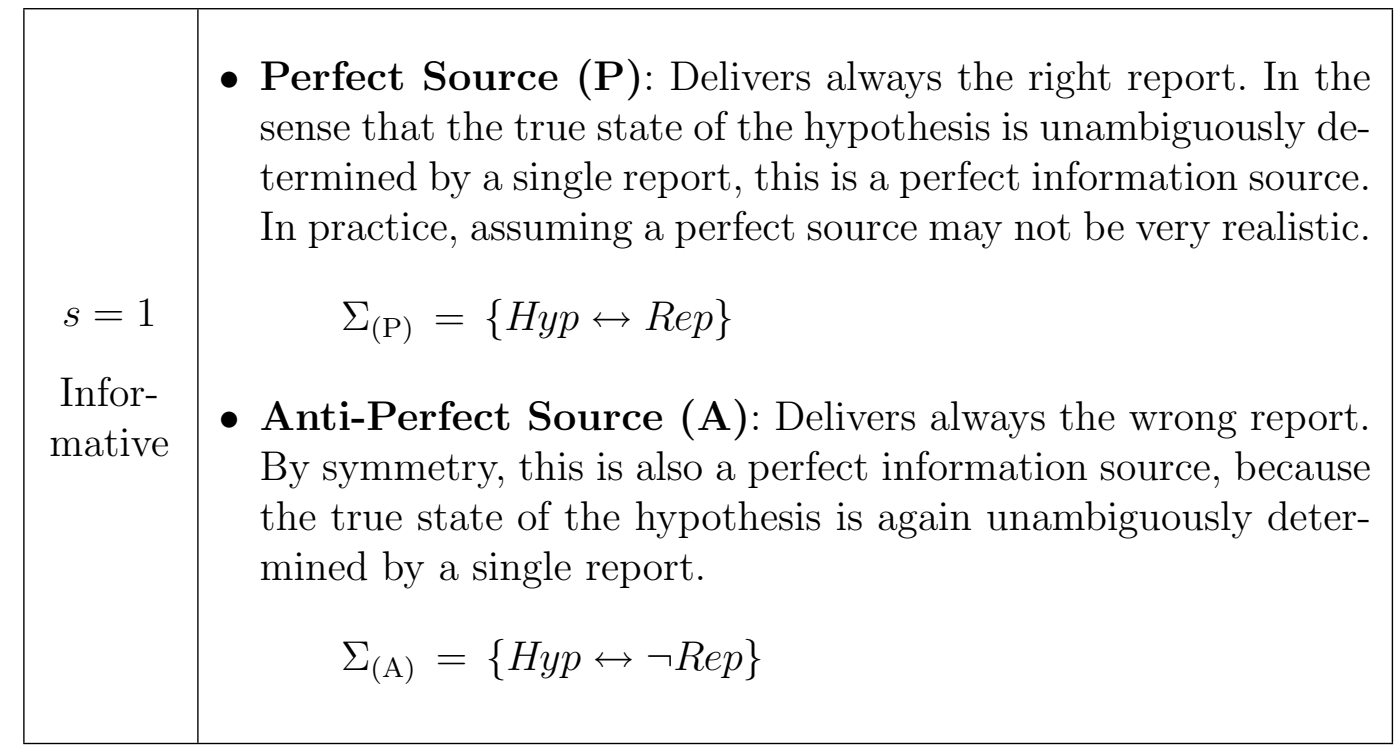

In the non-informative case of $V=\{R, R e p\}$, the report is supposed to depend on a single random event $R$ with a given prior probability $r=\operatorname{Pr}(R)$. In analogy to the models $(\mathrm{P})$ and $(\mathrm{A})$, that is by replacing Hyp by $R$, we obtain two non-informative models $(R)$ and $\left(R^{\prime}\right)$, respectively. Since they are perfectly symmetric with respect to $R$ and $\neg R$, only one of them is needed. Let's skip $\left(\mathrm{R}^{\prime}\right)$ and focus on $(\mathrm{R})$ :

$s=1 \quad$ - Randomizer (R): Delivers its report according to the outcome Non- $\quad$ of a random event $R$ with probability $r=\operatorname{Pr}(R)$.

Informative

$$
\Sigma_{(\mathrm{R})}=\{R \leftrightarrow R e p\}
$$

If we switch to $s=2$, the situation gets a bit more complicated. In the noninformative case of $V=\{P, Q, R e p\}$, i.e. if we suppose the reports to be influenced by two independent random events $P$ and $Q$ with corresponding prior probabilities $p=\operatorname{Pr}(P)$ and $q=\operatorname{Pr}(Q)$, it is easy to see that every model of that kind is reducible to the $(\mathrm{R})$ model. The idea is to identify the disjunction of configurations for which we get positive reports with $R$, and its complement with $\neg R$ (or vice versa). Note that this works for all noninformative models with $s \geq 2$. Here it means that only the informative case of $V=\{H y p, R, R e p\}$ is relevant.

Interesting informative models are the ones that are obtained from mixing the randomizer model $(\mathrm{R})$ with $(\mathrm{Y})$ and $(\mathrm{N})$. There are four such alternatives: 


\begin{tabular}{|c|c|}
\hline mative & $\begin{array}{l}\text { - Yes-Man/Randomizer (YR): If the hypothesis is true, the } \\
\text { report is always positive, otherwise it is generated at random. } \\
\text { Test functions of probabilistic algorithms are of that kind (see } \\
\text { Section 1). } \\
\qquad \Sigma_{(\mathrm{YR})}=\left\{\begin{array}{c}H y p \rightarrow R e p \\
\neg H y p \rightarrow(R \leftrightarrow R e p)\end{array}\right\} \\
\text { - Randomizer/Yes-Man (RY): If the hypothesis is true, the } \\
\text { report is generated at random, otherwise it is positive. This is } \\
\text { the inverse of the (YR) model. } \\
\qquad \Sigma_{(\mathrm{RY})}=\left\{\begin{array}{c}H y p \rightarrow(R \leftrightarrow R e p) \\
\neg H y p \rightarrow R e p\end{array}\right\} \\
\text { - Naysayer/Randomizer (NR): If the hypothesis is true, the } \\
\text { report is always negative, otherwise it is generated at random. } \\
\text { This is the negative counterpart of the (YR) model. } \\
\text { - Randomizer/Yes-Man (RN): If the hypothesis is true, the } \\
\text { report is generated at random, otherwise it is negative. This is } \\
\text { the inverse of the (NR) model and the negative counterpart of } \\
\text { the (NR) model. } \Sigma_{(\mathrm{NR})}=\left\{\begin{array}{c}H y p \rightarrow \neg R e p \\
\neg H y p \rightarrow(R \leftrightarrow R e p)\end{array}\right\} \\
\Sigma_{(\mathrm{RN})}=\left\{\begin{array}{c}H y p \rightarrow(R \leftrightarrow R e p) \\
\neg H y p \rightarrow \neg R e p\end{array}\right\}\end{array}$ \\
\hline
\end{tabular}

Another interesting mixed model is the one obtained from combining $(\mathrm{R})$ with $\left(\mathrm{R}^{\prime}\right)$. Logically, this is equivalent to a source who knows the true state of the hypothesis, but who chooses to give the correct report only under certain circumstances. This seems to be the more intuitive view, and we can look at it as the result of mixing the $(\mathrm{P})$ and $(\mathrm{A})$ models. It corresponds to the third approach discussed in Section 1 (Laplace, Condorcet, Boole, etc.), and as we will see in Section 4, it leads to a formula equivalent to (3). As an example, one can think of a defendant in court who denies being guilty. 


\begin{tabular}{|c|l|}
\hline \multirow{3}{*}{$s=2$} & $\begin{array}{l}\text { - Occasional Liar }(\mathbf{L}) \text { : Delivers the right report only under } \\
\text { certain random circumstances, that is according to a random } \\
\text { event } R \text { with probability } r=\operatorname{Pr}(R) \text {. Because the true state of } \\
\text { the hypothesis is known, this is a source that behaves like an } \\
\text { Infor- } \\
\text { occational liar. }\end{array}$ \\
$\Sigma_{(\mathrm{L})}=\{H y p \leftrightarrow(R \leftrightarrow R e p)\} \equiv\{R \leftrightarrow($ Hyp $\leftrightarrow$ Rep $)\}$
\end{tabular}

Before looking at the case $s=3$, let's recapitulate the models analyzed up to this point. The following table lists all possible configurations of positive or negative reports for $s=2$ and $V=\{H y p, R, R e p\}$. The total number of such configurations is $2^{2^{2}}=16$, which corresponds to the total number of complete possible models $c(2)=16$. It turns out that in each of these cases, we have either one of the 10 models already discussed or a symmetric one (with respect to $R$ and $\neg R$ ). In this sense, our taxonomy of complete models is exhaustive for $s \leq 2$.

\begin{tabular}{|c|c|c|c|c|c|}
\hline$H y p \wedge R$ & $H y p \wedge \neg R$ & $\neg H y p \wedge R$ & $\neg H y p \wedge \neg R$ & & \\
\hline$R e p$ & $\operatorname{Rep}$ & $\operatorname{Rep}$ & $R e p$ & $\Rightarrow$ & $(\mathrm{Y})$ \\
\hline $\operatorname{Rep}$ & Rep & $\operatorname{Rep}$ & $\neg R e p$ & $\Rightarrow$ & $(\mathrm{YR})$ \\
\hline $\operatorname{Rep}$ & $\operatorname{Rep}$ & $\neg R e p$ & $\operatorname{Rep}$ & $\Rightarrow$ & symmetric to (YR) \\
\hline Rep & Rep & $\neg R e p$ & $\neg R e p$ & $\Rightarrow$ & $(\mathrm{P})$ \\
\hline $\operatorname{Rep}$ & $\neg R e p$ & $\operatorname{Rep}$ & $\operatorname{Rep}$ & $\Rightarrow$ & $(\mathrm{RY})$ \\
\hline Rep & $\neg R e p$ & Rep & $\neg R e p$ & $\Rightarrow$ & $(\mathrm{R})$ \\
\hline $\operatorname{Rep}$ & $\neg R e p$ & $\neg R e p$ & $\operatorname{Rep}$ & $\Rightarrow$ & $(\mathrm{L})$ \\
\hline $\operatorname{Rep}$ & $\neg R e p$ & $\neg R e p$ & $\neg R e p$ & $\Rightarrow$ & $(\mathrm{RN})$ \\
\hline$\neg R e p$ & $\operatorname{Rep}$ & $\operatorname{Rep}$ & $\operatorname{Rep}$ & $\Rightarrow$ & symmetric to (RY) \\
\hline$\neg R e p$ & $\operatorname{Rep}$ & $\operatorname{Rep}$ & $\neg R e p$ & $\Rightarrow$ & symmetric to $(\mathrm{L})$ \\
\hline$\neg R e p$ & $\operatorname{Rep}$ & $\neg R e p$ & $\operatorname{Rep}$ & $\Rightarrow$ & symmetric to $(\mathrm{R})$ \\
\hline$\neg R e p$ & $\operatorname{Rep}$ & $\neg R e p$ & $\neg R e p$ & $\Rightarrow$ & symmetric to $(\mathrm{RN})$ \\
\hline$\neg R e p$ & $\neg R e p$ & $\operatorname{Rep}$ & $\operatorname{Rep}$ & $\Rightarrow$ & $(\mathrm{A})$ \\
\hline$\neg R e p$ & $\neg R e p$ & $\operatorname{Rep}$ & $\neg R e p$ & $\Rightarrow$ & $(\mathrm{NR})$ \\
\hline$\neg R e p$ & $\neg R e p$ & $\neg R e p$ & Rep & $\Rightarrow$ & symmetric to $(\mathrm{NR})$ \\
\hline$\neg R e p$ & $\neg R e p$ & $\neg R e p$ & $\neg R e p$ & $\Rightarrow$ & $(\mathrm{N})$ \\
\hline
\end{tabular}

For $s=3$, a comprehensive discussion of all $c(3)=256$ complete models would go beyond the scope of this paper. In this subsection, we will only add one more model, the one that, as we will see, includes all previously discussed models as special cases. Further complete models for $s=3$ will be discussed in Subsection 3.4. 
The idea is to mix the $(\mathrm{R})$ model with itself, but instead of considering a single random event $R$, we suppose the report to depend on two distinct and independent random events $P$ and $Q$, coinciding with $P$ in case of $H y p$ and with $Q$ in case of $\neg H y p$. In other words, false positives are produced by $\neg P$ and false negatives by $Q$. For $p=\operatorname{Pr}(P)$ and $q=\operatorname{Pr}(Q)$, this model corresponds to the first Bayesian network discussed in Section 1.

\begin{tabular}{|c|c|}
\hline$s=3$ & $\begin{array}{l}\text { - Indicator (D): Depending on whether Hyp is true or false, the } \\
\text { correct report is only delivered under certain circumstances } P \\
\text { and } \neg Q \text {, respectively. As an example, one can think of a medical } \\
\text { test producing false positives and false negatives, respectively. }\end{array}$ \\
$\begin{array}{c}\text { Infor- } \\
\text { mative }\end{array}$ & $\left.\begin{array}{c}H y p \rightarrow(P \leftrightarrow R e p) \\
\neg H y p \rightarrow(Q \leftrightarrow R e p)\end{array}\right\}$ \\
\hline
\end{tabular}

Figure 3 (see below) summarizes all complete models discussed in this subsection. The (D) model is in the center and contains all other models as special cases. For example, if $P$ is always true $(p=1)$, and by renaming $Q$ by $R$, we see that (D) degenerates into (YR). The (YR) model itself degenerates into $(\mathrm{Y})$ and $(\mathrm{P})$, if $R$ is either true $(r=1)$ or false $(r=0)$, respectively. Another example is the $(\mathrm{L})$ model, which degenerates from (D) whenever $P$ and $Q$ exclude each other (logically speaking, for $P \leftrightarrow \neg Q$ ). From (L), if $R$ is either true or false, we get $(\mathrm{P})$ and $(\mathrm{A})$, respectively.

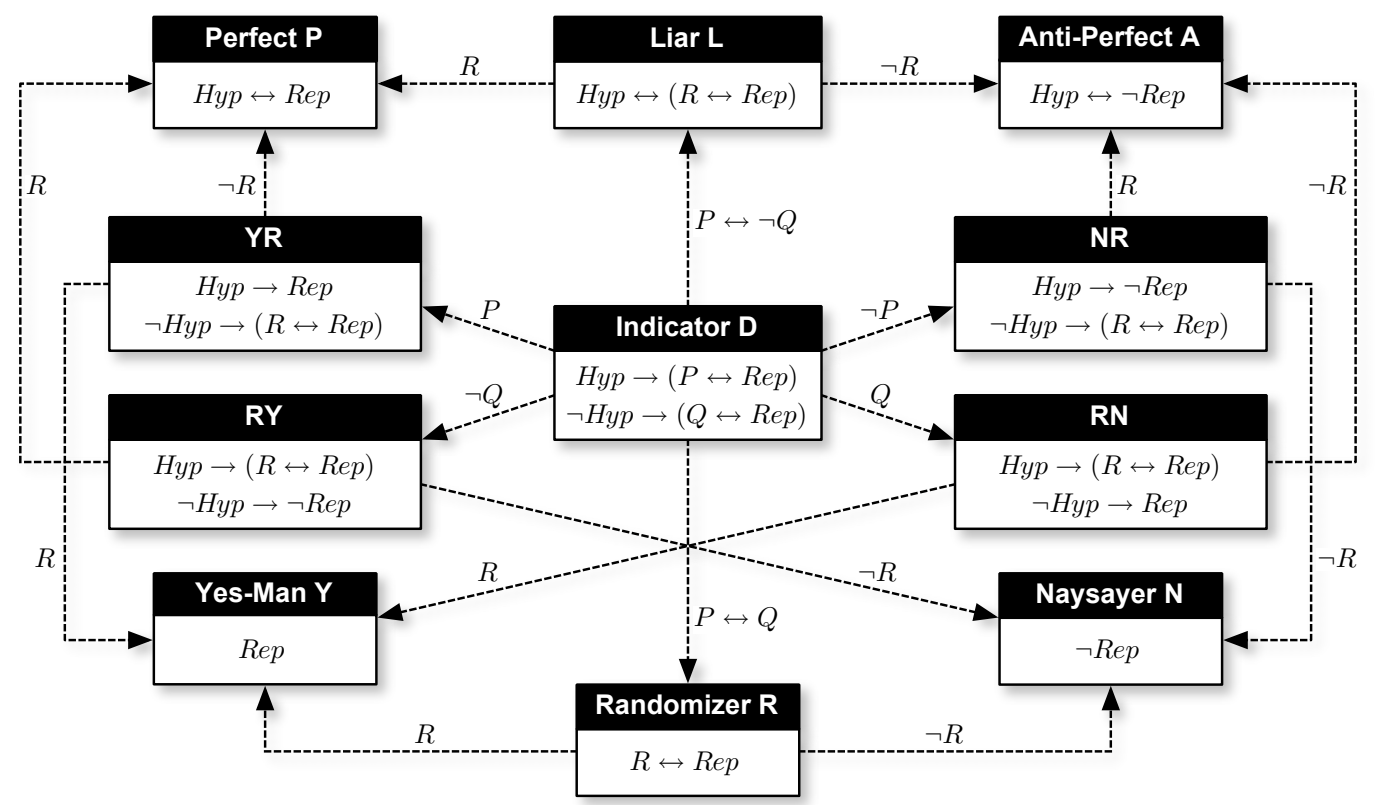

Fig. 3. The taxonomy of complete models. A dotted arrow from one model to another means that the latter is a special case of the former. 


\subsection{Incomplete Models}

Let's now turn our attention to incomplete models. Recall that the total number of incomplete models is $i(c)=3^{2^{s}}-2^{2^{s}}$, i.e. $i(0)=1, i(1)=5, i(2)=65$, and so on. Again, many of them are equivalent up to symmetry, and they need not all to be mentioned explicitly. Furthermore, we will not consider cases in which the positive (negative) outcome of a random event $R$ is known to generate a positive (negative) report, but not the other way round. In other words, we will not discuss models that are incomplete with respect to random events $R$, for example $\Sigma_{(. .)}=\{R \rightarrow \operatorname{Rep}\}, \Sigma_{(. .)}=\{H y p \rightarrow(R \rightarrow R e p)\}$, or likewise. In the next subsection, we will look at some of them from a different angle.

In the simplest case of $s=0$, only one model is possible. It stands for the case in which absolutely nothing is known about the source. This is the most trivial model among all, and it does not allow any meaningful conclusions. In other words, reports obtained from such an unknown source are of no value with respect to Hyp.

$s=0$

- Ignorant (I): Nothing is known about the source, except that

Noneither a positive or a negative report is delivered.

Informative

$$
\Sigma_{(\mathrm{I})}=\{\operatorname{Rep} \vee \neg \operatorname{Rep}\} \equiv\{\top\} \equiv\{\}
$$

For $s=1$ and $V=\{H y p, R e p\}$, we have $i(1)=5$ incomplete models, but one of them degenerates into the non-informative model (I). The four remaining informative models are obtained from mixing (I) with the models $(\mathrm{Y})$ and $(\mathrm{N})$, respectively.

\begin{tabular}{|c|c|}
\hline $\begin{array}{l}s=1 \\
\text { Infor- } \\
\text { mative }\end{array}$ & $\begin{array}{l}\text { - Yes-Man/Ignorant (YI): If the hypothesis is true, the report } \\
\text { is known to be always positive, but nothing is known about the } \\
\text { case } \neg H y p \text {. As an example, one can think of a probabilistic } \\
\text { algorithm and a corresponding test with an unknown ratio of } \\
\text { false negatives. } \\
\qquad \Sigma_{(\mathrm{YI})}=\{H y p \rightarrow R e p\} \\
\text { - Ignorant/Yes-Man (IY): If the hypothesis is false, the report } \\
\text { is known to be always positive, but nothing is known about the } \\
\text { case } H y p \text {. } \\
\qquad \Sigma_{(\mathrm{IY})}=\{\neg H y p \rightarrow R e p\}\end{array}$ \\
\hline
\end{tabular}




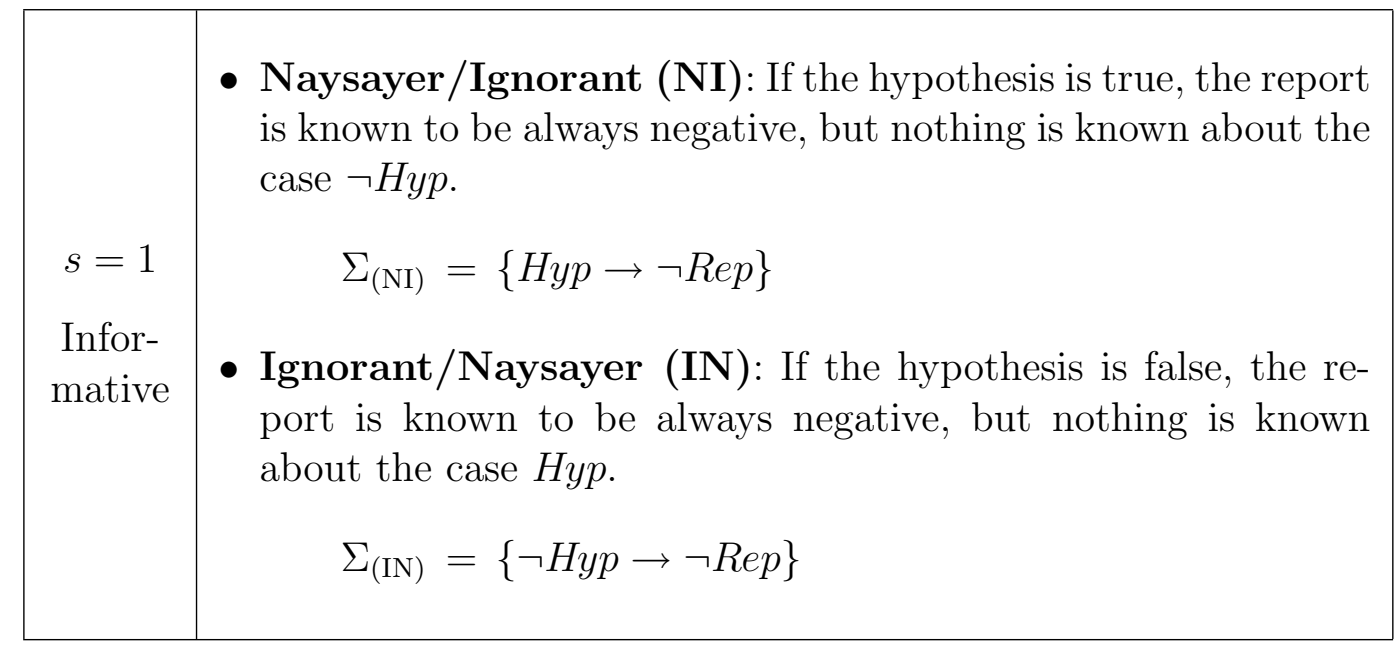

Numerous incomplete models exist for $s=2$ and $V=\{H y p, R, R e p\}$. The most interesting ones are obtained from mixing the models (I) and (R). There are two such alternatives, each of them corresponding to one "half" of the complete model (D). Hence, they represent situations in which either the ratio of false positives or the ratio of false negatives is known, but not both of them at same time.

\begin{tabular}{|c|c|}
\hline $\begin{array}{l}s=2 \\
\text { Infor- } \\
\text { mative }\end{array}$ & $\begin{array}{l}\text { - Randomizer/Ignorant (RI): If the hypothesis is true, the } \\
\text { report is known to be generated at random, but nothing is } \\
\text { known about the case } \neg H y p \text {. As an example, on can think of a } \\
\text { medical test for which the ratio of false positives is known, but } \\
\text { with an unknown ratio of false negatives. } \\
\qquad \Sigma_{(\mathrm{RI})}=\{H y p \rightarrow(R \leftrightarrow R e p)\} \\
\text { - Ignorant/Randomizer (IR): If the hypothesis is false, the } \\
\text { report is known to be generated at random, but nothing is } \\
\text { known about the case } H y p \text {. As an example, on can think of a } \\
\text { medical test for which the ratio of false negatives is known, but } \\
\text { with an unknown ratio of false positives. } \\
\qquad \Sigma_{(\mathrm{IR})}=\{\neg H y p \rightarrow(R \leftrightarrow R e p)\}\end{array}$ \\
\hline
\end{tabular}

Figure 4 gives an overview of all incomplete model discussed up to this point. It indicates that the models (YI) and (NY) are special cases of (RI), whereas (IY) and (IN) are special cases of (IR). The (I) model is the least complete one among all. Recall that the taxonomy shown in Fig. 4 is not exhaustive and restricted to $s \leq 2$. Further (complete and) incomplete models will be discussed in the following subsection. 


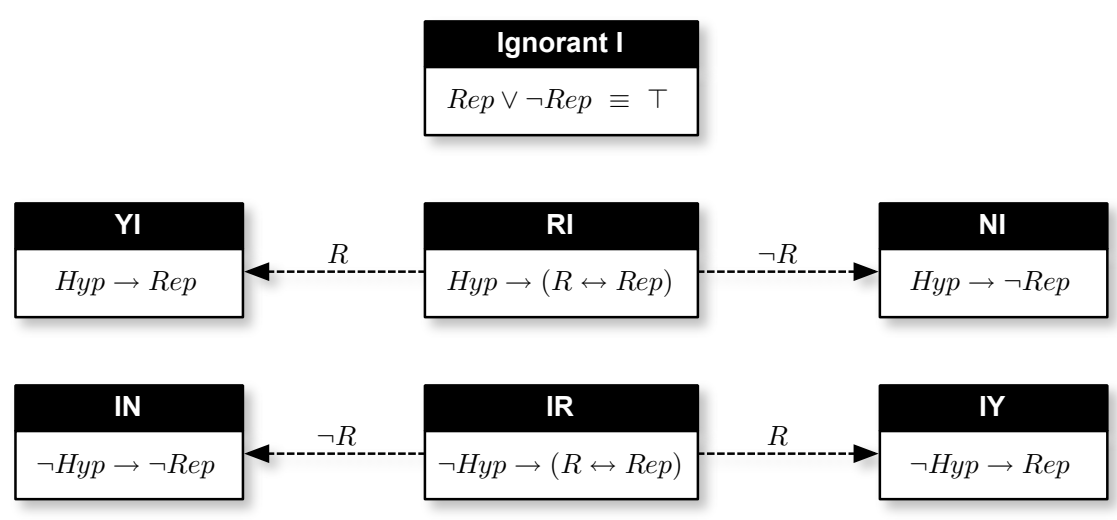

Fig. 4. The taxonomy of incomplete models. A dotted arrow from one model to another means that the latter is a special case of the former.

\subsection{Reliability-Based Models}

Based on the complete and incomplete models introduced in the previous two subsections, we will now show how to build so-called reliability-based models. The idea is similar to the second approach in Section 1, which is based on a reliability variable $R E L$ with $R e l$ and $\neg$ Rel as possible values. One can think of two distinct operating modes, one always producing the right report, the other one not. The respective probabilities are $\rho=\operatorname{Pr}(\operatorname{Rel})$ and $\bar{\rho}=1-\rho=$ $\operatorname{Pr}(\neg \operatorname{Rel})$. We will always suppose $R E L$ to be probabilistically independent of any other random event like $P, Q$, or $R$.

In the rest of the paper, we will assume that a reliable source behaves in an ideal or perfect way, that is according to the $(\mathrm{P})$ model. Note that not only the $(\mathrm{P})$ but also the $(\mathrm{A})$ model, by symmetry, behaves perfectly. All other previously discussed models are imperfect. We may thus combine the two perfect models $(\mathrm{P})$ and $(\mathrm{A})$ with the sixteen imperfect ones. Our convention is to write $(\mathrm{PM})$ and $(\mathrm{AM})$ for the respective combinations of $(\mathrm{P})$ and $(\mathrm{A})$ with any imperfect model $(\mathrm{M})$. Formally, if $\Sigma_{(\mathrm{M})}$ is the set of sentences of an imperfect model $(\mathrm{M})$, and $\sigma_{(\mathrm{M})}=\wedge \Sigma_{(\mathrm{M})}$ the corresponding conjunction of sentences, then the models (PM) and (AM) are by

$$
\Sigma_{(\mathrm{PM})}=\left\{\begin{array}{l}
\operatorname{Rel} \rightarrow(\text { Hyp } \leftrightarrow \text { Rep }) \\
\neg \operatorname{Rel} \rightarrow \sigma_{(\mathrm{M})}
\end{array}\right\}, \quad \Sigma_{(\mathrm{AM})}=\left\{\begin{array}{l}
\operatorname{Rel} \rightarrow(\text { Hyp } \leftrightarrow \neg R e p) \\
\neg \operatorname{Rel} \rightarrow \sigma_{(\mathrm{M})}
\end{array}\right\}
$$

Some of the reliability-based models are more interesting than others. In general, one can say that the (PM) models are more interesting than the (AM) models, because the latter are obtained from the former by symmetry. In the 
following, our focus will be on the (PM) models. Note that any combination of (P) with a complete (incomplete) model is again complete (incomplete). Furthermore, it is clear that all reliability-based models models are informative with respect to the hypothesis.

Let us first look at possible complete models. Recall that our taxonomy of complete models is exhaustive for $s \leq 2$ (up to symmetry). Consequently, by combining $(\mathrm{P})$ with the two trivial models $(\mathrm{Y})$ and $(\mathrm{N})$, we have $s=2$, i.e. we must obtain two previously discussed complete models. In fact, it is easy to see that $(\mathrm{PY})$ is logically equivalent to $(\mathrm{YR})$, whereas $(\mathrm{PN})$ is equivalent to (RY). Similarly, if (A) instead of (P) is used as perfect model, we get logically equivalent counterparts for $(\mathrm{NR})$ and $(\mathrm{RN})$, respectively. Another possible combination, namely the (PL) model, drops out, because by substituting Rel $\mathrm{V}$ $R$ by another random event $R^{\prime}$ with $r^{\prime}=\operatorname{Pr}\left(R^{\prime}\right)=1-\bar{\rho} \bar{r}$, it is reducible to a simple (L) model.

Among the six remaining models, the most interesting ones are (PR) and (PD). The former is characterized by $V=\{H y p, \operatorname{Rel}, R, \operatorname{Rep}\}$ and thus $s=3$. With $V=\{H y p, \operatorname{Rel}, P, Q, \operatorname{Rep}\}$ and $s=4$, the (PD) model is the most general and accurate one among all complete models discussed in this paper. A full model specification for (PD) consists of three parameters $\rho, p$, and $q$, which corresponds to the number of parameters in the general model of Section 2 . We have thus reached the maximal level of generality, that is models with $s=4$ or beyond need not to be considered.

\begin{tabular}{|c|c|}
\hline$s=3$ & $\begin{array}{l}\text { - Perfect/Randomizer (PR): If the source happens to be unre- } \\
\text { liable, the report is generated at random. The second model of } \\
\text { Section } 1 \text { is of that kind. } \\
\qquad \Sigma_{(\mathrm{PR})}=\left\{\begin{array}{c}\operatorname{Rel} \rightarrow(\text { Hyp } \leftrightarrow R e p) \\
\neg \operatorname{Rel} \rightarrow(R \leftrightarrow R e p)\end{array}\right\}\end{array}$ \\
\hline$s=4$ & $\begin{array}{l}\text { - Perfect/Indicator (PD): If the source happens to be unreli- } \\
\text { able, it behaves like an indicator }(\mathrm{D}) \text {, i.e. it may generate false } \\
\text { positives and false negatives. } \\
\qquad \Sigma_{(\mathrm{PD})}=\left\{\begin{array}{c}\operatorname{Rel} \rightarrow(H y p \leftrightarrow R e p) \\
\neg \operatorname{Rel} \rightarrow(H y p \rightarrow(P \leftrightarrow R e p)) \\
\neg \operatorname{Rel} \rightarrow(\neg H y p \rightarrow(Q \leftrightarrow R e p))\end{array}\right\}\end{array}$ \\
\hline
\end{tabular}

Among the possible combinations obtained from incomplete models, (PI) is the simplest and most important one. The following discussion will be restricted to it. 
The (PI) model represents a situation in which the behavior of the source is only partially known. As an example, one can think of a technical sensor with various unknown failure modes. In case of $\operatorname{Rel}$ with probability $\rho$, that is if the sensor works properly, it produces the correct report, but nothing is known about the report (and therefore about the hypothesis) in case of $\neg$ Rel. We can look at the (PI) model as the "upper half" of the complete model (L).

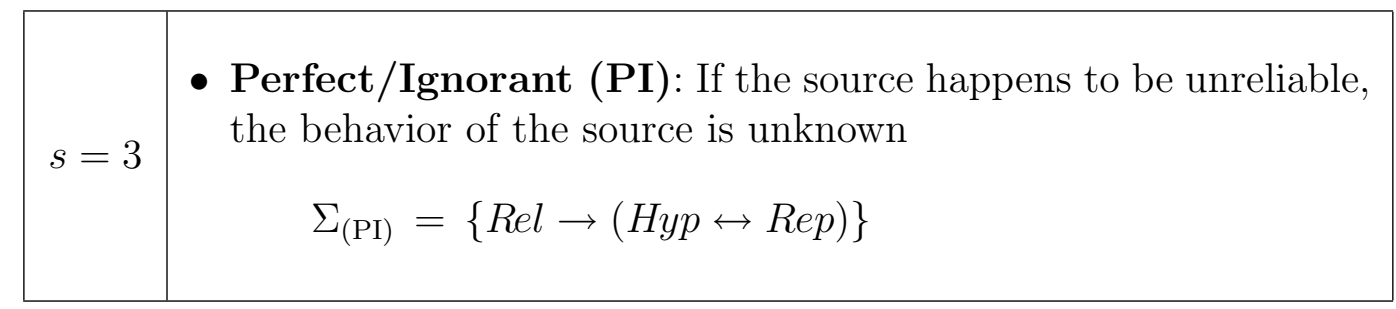

As we will see in Section 4, the lack of knowledge of an incomplete model will lead to different values for $d s p(\{H y p\})$ and $d p s(\{H y p\})$. In general, the amount of lacking information is quantified by $d p s(\{H y p\})-d s p(\{H y p\})$, a quantity sometimes called degree of ignorance [17].

\subsection{Computing the Parameters}

Now let our attention be directed to the determination of the parameters $x_{1}$, $y_{1}, z_{1}$ for positive reports and $x_{2}, y_{2}, z_{2}$ for negative reports. The problem is to transform the logical description of the various models into corresponding mass functions. A general solution of this transformation problem is described in [16]. The common frame of discernment of all possible models is the Cartesian product

$$
\begin{aligned}
\Theta & =H Y P \times R E P \\
& =\{H y p \wedge \operatorname{Rep}, H y p \wedge \neg \operatorname{Rep}, \neg H y p \wedge \operatorname{Rep}, \neg H y p \wedge \neg \operatorname{Rep}\} .
\end{aligned}
$$

For illustrative purposes, we will focus on the discussion of the (PR) model. All other models can be analyzed in an analogous way. At the end of this subsection, we will summarize all other results as a theorem.

The (PR) model contains two randomization parameters $\rho$ and $r$. Because we assume the corresponding events $R e l$ and $R$ to be probabilistically independent (according to the remark in the previous subsection), there are four possible combined events $\operatorname{Re} l \wedge R, \operatorname{Re} l \wedge \neg R, \neg \operatorname{Rel} \wedge R, \neg \operatorname{Rel} \wedge \neg R$ with probabilities $\rho r$, $\rho \bar{r}, \bar{\rho} r, \bar{\rho} \bar{r}$, respectively. In the context of probabilistic argumentation, these combined events are called scenarios $[15,17]$. 
Each of the scenarios has certain consequences for the possible true state of $\Theta$. Let $\Gamma_{\mathrm{PR}}(\mathbf{s})$ denote the set of possible states of $\Theta$ if scenario $\mathbf{s}$ is assumed to be the true one. In the (PR) model, which is defined by two logical statements $R e l \rightarrow(H y p \leftrightarrow R e p)$ and $\neg \operatorname{Rel} \rightarrow(R \leftrightarrow R e p)$, this means that

$$
\begin{aligned}
\Gamma_{\mathrm{PR}}(\operatorname{Rel} \wedge R) & =\{H y p \wedge \text { Rep }, \neg H y p \wedge \neg \operatorname{Rep}\}, \\
\Gamma_{\mathrm{PR}}(\operatorname{Rel} \wedge \neg R) & =\{H y p \wedge \text { Rep }, \neg H y p \wedge \neg \operatorname{Rep}\}, \\
\Gamma_{\mathrm{PR}}(\neg \operatorname{Rel} \wedge R) & =\{H y p \wedge \text { Rep, } \neg H y p \wedge \operatorname{Rep}\}, \\
\Gamma_{\mathrm{PR}}(\neg \operatorname{Rel} \wedge \neg R) & =\{H y p \wedge \neg \operatorname{Rep}, \neg H y p \wedge \neg \operatorname{Rep}\} .
\end{aligned}
$$

Together with the probabilities indicated above, we obtain a mass function $m_{\text {PR }}$ over $\Theta$. The only non-zero masses are

$$
\begin{aligned}
m_{\mathrm{PR}}(\{H y p \wedge \operatorname{Rep}, \neg H y p \wedge \neg \operatorname{Rep}\}) & =\rho r+\rho \bar{r}=\rho, \\
m_{\mathrm{PR}}(\{H y p \wedge \operatorname{Rep}, \neg H y p \wedge \operatorname{Rep}\}) & =\bar{\rho} r \\
m_{\mathrm{PR}}(\{H y p \wedge \neg \operatorname{Rep}, \neg H y p \wedge \neg \operatorname{Rep}\}) & =\bar{\rho} \bar{r} .
\end{aligned}
$$

Now suppose that the information source provides a positive report. The above mass function has then to be conditioned on Rep. The result is a new mass function $m_{\mathrm{PR}}^{+}$over $\Theta$ with

$$
\begin{aligned}
m_{\mathrm{PR}}^{+}(\{H y p \wedge \operatorname{Rep}\}) & =\rho, \\
m_{\mathrm{PR}}^{+}(\{H y p \wedge \operatorname{Rep}, \neg H y p \wedge \operatorname{Rep}\}) & =\bar{\rho} r, \\
m_{\mathrm{PR}}^{+}(\emptyset) & =\bar{\rho} \bar{r} .
\end{aligned}
$$

Finally, projecting $m_{\mathrm{PR}}^{+}$from the original frame of discernment $\{H Y P, R E P\}$ to the new frame $\{H Y P\}$ means to replace $\{H y p \wedge R e p\}$ by $\{H y p\}$ and $\{H y p \wedge$ $R e p, \neg H y p \wedge R e p\}$ by $\{H y p, \neg H y p\}$. The result of this is a mass function $m_{\mathrm{PR}}^{+}$ over $H Y P$ with

$$
\begin{aligned}
m_{\mathrm{PR}}^{+}(\emptyset) & =\bar{\rho} \bar{r}, \\
m_{\mathrm{PR}}^{+}(\{H y p\}) & =\rho, \\
m_{\mathrm{PR}}^{+}(\{\neg H y p\}) & =0, \\
m_{\mathrm{PR}}^{+}(\{H y p, \neg H y p\}) & =\bar{\rho} r .
\end{aligned}
$$


According to the definition of the parameters $x_{i}, y_{i}$, and $z_{i}$ in Subsection 2.1, this implies

$$
\begin{aligned}
& x_{1}=\rho+\bar{\rho} r=1-\bar{\rho} \bar{r}, \\
& y_{1}=0+\bar{\rho} r=\bar{\rho} r, \\
& z_{1}=\bar{\rho} r,
\end{aligned}
$$

which are the three parameters for positive reports in the $(\mathrm{PR})$ model. In the case of a negative report, the analysis is analogous. We derive from $m_{\mathrm{PR}}$ a new mass function $m_{\mathrm{PR}}^{-}$over $\Theta$ with

$$
\begin{aligned}
m_{\mathrm{PR}}^{-}(\{\neg H y p \wedge \neg \operatorname{Rep}\}) & =\rho, \\
m_{\mathrm{PR}}^{-}(\emptyset) & =\bar{\rho} r, \\
m_{\mathrm{PR}}^{-}(\{H y p \wedge \neg \operatorname{Rep}, \neg H y p \wedge \neg \operatorname{Rep}\}) & =\bar{\rho} \bar{r} .
\end{aligned}
$$

Projecting $m_{\mathrm{PR}}^{-}$in the same way as above from $\{H Y P, R E P\}$ to $\{H Y P\}$ then leads to

$$
\begin{aligned}
m_{\mathrm{PR}}^{+}(\emptyset) & =\bar{\rho} r, \\
m_{\mathrm{PR}}^{+}(\{H y p\}) & =0, \\
m_{\mathrm{PR}}^{+}(\{\neg H y p\}) & =\rho, \\
m_{\mathrm{PR}}^{+}(\{H y p, \neg H y p\}) & =\bar{\rho} \bar{r},
\end{aligned}
$$

from which we obtain the parameters for negative reports in the (PR) model:

$$
\begin{aligned}
& x_{2}=0+\bar{\rho} \bar{r}=\bar{\rho} \bar{r}, \\
& y_{2}=\rho+\bar{\rho} \bar{r}=1-\bar{\rho} r, \\
& z_{2}=\bar{\rho} \bar{r} .
\end{aligned}
$$

A similar procedure works for all other models. In most cases, the analysis is at least as simple as it is here. We will provide all other results as a theorem. For more information about the transformation from logical models into mass function we refer to [16].

Theorem 2 The results shown in the following table are the parameters for the models introduced in the previous subsection. 


\begin{tabular}{|c|c|c|c|c|c|c|}
\hline \multirow[b]{2}{*}{ Model } & \multicolumn{3}{|c|}{ Positive Report } & \multicolumn{3}{|c|}{ Negative Report } \\
\hline & $x_{1}$ & $y_{1}$ & $z_{1}$ & $x_{2}$ & $y_{2}$ & $z_{2}$ \\
\hline$(\mathrm{Y})$ & 1 & 1 & 1 & 0 & 0 & 0 \\
\hline$(\mathrm{N})$ & 0 & 0 & 0 & 1 & 1 & 1 \\
\hline$(\mathrm{P})$ & 1 & 0 & 0 & 0 & 1 & 0 \\
\hline$(\mathrm{A})$ & 0 & 1 & 0 & 1 & 0 & 0 \\
\hline$(\mathrm{R})$ & $r$ & $r$ & $r$ & $\bar{r}$ & $\bar{r}$ & $\bar{r}$ \\
\hline$(\mathrm{YR})$ & 1 & $r$ & $r$ & 0 & $\bar{r}$ & 0 \\
\hline$(\mathrm{RY})$ & $r$ & 1 & $r$ & $\bar{r}$ & 0 & 0 \\
\hline$(\mathrm{NR})$ & 0 & $r$ & 0 & 1 & $\bar{r}$ & $\bar{r}$ \\
\hline$(\mathrm{RN})$ & $r$ & 0 & 0 & $\bar{r}$ & 1 & $\bar{r}$ \\
\hline$(\mathrm{L})$ & $r$ & $\bar{r}$ & 0 & $\bar{r}$ & $r$ & 0 \\
\hline (D) & $p$ & $q$ & $p q$ & $\bar{p}$ & $\bar{q}$ & $\bar{p} \bar{q}$ \\
\hline (I) & 1 & 1 & 1 & 1 & 1 & 1 \\
\hline$(\mathrm{YI})$ & 1 & 1 & 1 & 0 & 1 & 0 \\
\hline$(\mathrm{IY})$ & 1 & 1 & 1 & 1 & 0 & 0 \\
\hline$(\mathrm{NI})$ & 0 & 1 & 0 & 1 & 1 & 1 \\
\hline$(\mathrm{IN})$ & 1 & 0 & 0 & 1 & 1 & 1 \\
\hline$(\mathrm{RI})$ & $r$ & 1 & $r$ & $\bar{r}$ & 1 & $\bar{r}$ \\
\hline$(\mathrm{IR})$ & 1 & $r$ & $r$ & 1 & $\bar{r}$ & $\bar{r}$ \\
\hline$(\mathrm{PR})$ & $1-\bar{\rho} \bar{r}$ & $\bar{\rho} r$ & $\bar{\rho} r$ & $\bar{\rho} \bar{r}$ & $1-\bar{\rho} r$ & $\bar{\rho} \bar{r}$ \\
\hline$(\mathrm{PD})$ & $1-\bar{\rho} \bar{p}$ & $\bar{\rho} q$ & $\bar{\rho} p q$ & $\bar{\rho} \bar{p}$ & $1-\bar{\rho} q$ & $\bar{\rho} \bar{p} \bar{q}$ \\
\hline$(\mathrm{PI})$ & 1 & $\bar{\rho}$ & $\bar{\rho}$ & $\bar{\rho}$ & 1 & $\bar{\rho}$ \\
\hline
\end{tabular}

For lack of space, we abstain from giving an explicit proof of the above theorem and refer to the discussion earlier in this subsection. In Section 4, we will analyze the most interesting models by inserting the corresponding parameters into (10) and (11).

\subsection{Modeling Prior Knowledge}

Prior knowledge, if available, is an important factor to be considered. It is usually the product of previous reports from partially reliable sources that are not further specified. Or it may simply reflect a personal opinion, sentiment, 
or prejudice regarding the true state of $H Y P$. We will not further take the origin of the prior knowledge into account. This means that $H Y P$ is the only variable to be considered. As a consequence, we can represent prior knowledge as a mass function over $H Y P$ specified by parameters $x, y$, and $z$ as explained before.

We have shown in (10) and (11) how the presence of prior knowledge over $H Y P$ influences the overall judgement of $H Y P$. And we have already discussed two special cases of $z=0$ (a prior probability $h=\operatorname{Pr}(H y p)$ is given) and $z=1$ (no prior knowledge available). In order to get a proper interpretation of these parameters, i.e. an interpretation that is applicable to the general case of $0 \leq z \leq 1$, consider the following two models $(\mathrm{C})$ and $(\mathrm{V})$ :

- Confidence (C): Let there be a prior distribution over HYP in which one is more or less confident. This means that in some cases, the prior distribution happens to be a reasonable one that is built on solid ground, but it may also be an arbitrary result of some misinformation. Suppose that the strength of the confidence is expressed by the probability $\gamma=\operatorname{Pr}($ Con $)$ of an event Con. We can then look at Hyp as an event which depends primarily on Con and secondarily on a random event $H$ with $h=\operatorname{Pr}(H)$. Logically, this can be expressed by

$$
\text { Con } \rightarrow(H \leftrightarrow H y p) .
$$

Note that nothing is said about Hyp in case of $\neg$ Con. As an example, think of flipping a coin that has been drawn at random from a bag that initially contained two fair and one unknown coin. This particular case could be represented by $\gamma=\frac{2}{3}$ and $h=\frac{1}{2}$.

- Interval-Valued Probabilities (V): Suppose that it is not possible to specify a prior distribution over $H Y P$ precisely, but let there be an interval probability $[\ell, u]$ that specifies lower and upper bounds for a precise value of $h=\operatorname{Pr}($ Hyp $)$. In other words, we assume to have two parameters $\ell$ and $u$ with $\ell \leq h \leq u$ instead of a single parameter $h$.

According to [22], imprecise probabilities may have various sources. Most intuitively, one could say that the impreciseness is due to some lack of information. In this sense, the degree of impreciseness $u-l$ reflects the amount of available prior knowledge. Clearly, $[0,1]$ and $[h, h]$ are the opposite extreme cases of total ignorance and a precise parameter $h$, respectively. In the context of probabilistic argumentation or DST, it is also possible to consider the results obtained from previously evaluating reports from partially reliable information sources as probability intervals [ $\operatorname{sp}(\operatorname{Hyp}), d p s(H y p)]$.

Both models (C) and (V) correspond to a situation in which the holder of the prior knowledge is not totally sure about the opinion or feeling she or he has about Hyp. In practice, such a situation seems to be rather typical than 
exceptional. The parameters $x, y$, and $z$ for the general model are given in the following theorem. Note that both the $(\mathrm{C})$ and the $(\mathrm{V})$ model can be combined with any other model discussed in the previous section. For example, we will call $(\mathrm{CPI})$ the model that is obtained from the combination of the $(\mathrm{C})$ and the (PI) model.

Theorem 3 The results shown in the following table are the parameters for the models of prior knowledge introduced above.

\begin{tabular}{|c||c|c|c|}
\hline Model & $x$ & $y$ & $z$ \\
\hline \hline$(\mathrm{C})$ & $1-\gamma \bar{h}$ & $1-\gamma h$ & $\bar{\gamma}$ \\
\hline$(\mathrm{V})$ & $u$ & $\bar{\ell}$ & $u-\ell$ \\
\hline
\end{tabular}

Proof: The (C) model implies $\Gamma_{\mathrm{C}}($ Con $\wedge H)=\{H y p\}, \Gamma_{\mathrm{C}}($ Con $\wedge \neg H)=$ $\{\neg H y p\}$, and $\Gamma_{\mathrm{C}}(\neg C o n \wedge H)=\Gamma_{\mathrm{C}}(\neg$ Con $\wedge \neg H)=\{H y p, \neg H y p\}$ with probabilities $\gamma h, \gamma \bar{h}, \bar{\gamma} h$, and $\bar{\gamma} \bar{h}$, respectively. This means that $m_{\mathrm{C}}(\{H y p\})=\gamma h$, $m_{\mathrm{C}}(\{\neg H y p\})=\gamma \bar{h}$, and $m_{\mathrm{C}}(\{H y p, \neg H y p\})=\bar{\gamma}$, from which we can derive $x=\gamma h+\bar{\gamma}=1-\gamma \bar{h}, y=\gamma \bar{h}+\bar{\gamma}=1-\gamma h$, and $z=\bar{\gamma}$. In the (V) model, if the parameters $\ell$ and $u$ are interpreted as $d s p(H y p)$ and $d p s(H y p)$ of a previous evaluation, we have directly $m_{\mathrm{V}}(\{H y p\})=\ell, m_{\mathrm{V}}(\{\neg H y p\})=1-u$, and $m_{\mathrm{V}}(\{H y p, \neg H y p\})=u-\ell$. This implies $x=\ell+u-\ell=u, y=1-u+u-\ell=\bar{\ell}$, and $z=u-\ell$.

Note that the models $(\mathrm{C})$ and $(\mathrm{V})$ are not so much different as they appear to be at first sight. In fact, it is always possible to transform parameters $\gamma$ and $h$ unequivocally into parameters $\ell$ and $u$ and vice versa:

- from (C) to (V): $\ell=\gamma h, u=1-\gamma \bar{h}$,

- from $(\mathrm{V})$ to $(\mathrm{C}): \gamma=1-(u-\ell), h=\frac{\ell}{1-(u-\ell)}$.

Hence the (V) model can be simulated by the (C) model (and vice versa). To avoid redundancies and to keep things simple, we will restrict the following discussion to the $(\mathrm{C})$ model.

A pleasant property of the $(\mathrm{C})$ model is the possibility to vary the strength of the prior knowledge continuously between 0 and 1 with the parameter $\gamma$. The situation where no prior knowledge is available is represented by $\gamma=0$ and it implies $x=y=z=1$. On the other hand, if we consider a situation where a precise prior probability $h=\operatorname{Pr}($ Hyp $)$ is available, we have $\gamma=1$ and therefore $x=h, y=\bar{h}$, and $z=0$. This leads to the reduction of (10) and (11) to (12). 


\section{Case Studies}

The purpose of this section is to examine certain models of partially reliable sources more closely. A good candidate for a further analysis is the indicator model (D). As demonstrated earlier, (D) is the most general complete model of Fig. 3. Even more general is the (PD) model. Because of the practical difficulty to specify all of its three parameters, we prefer to analyze the $(\mathrm{PR})$ rather than the (PD) model. Another reason for this is the fact that the (PR) model makes a direct connection to one of the introductory examples in Section 1. For the same reason, we will also take a close look at the models (L) and (YR). Because of its simplicity, another interesting model is (PI).

In combination with the $(\mathrm{C})$ model for prior knowledge, we will talk about the (CPI), (CL), (CD), (CPR), or (CYR) model, respectively. The corresponding expressions for degree of support and degree of possibility are obtained by substituting the parameters in (10) and (11) by the respective values of Theorem 2 and 3 . Note that the general case of $0<\gamma<1$ does not allow significant simplifications. Our discussion will thus focus on the two extreme cases $\gamma=0$ and $\gamma=1$, which means that we can also derive the results from (12), (13), and (14).

We will use $\delta=n-m$ to denote the margin between positive and negative reports and $N=n+m$ for the total number of reports. In many practical applications one would expect the results to depend on both $\delta$ and $N$. For example, 10 positive and 0 negative reports seem to be a different situation than 1010 positive and 1000 negative reports $[2,7]$. We will see that some models give the same degrees of support and possibility for both situations.

\subsection{The Model (CPI)}

Consider the (PI) model in which the outcome of $R E P$ only depends on $R E L$. For $\gamma=0$ (no prior knowledge), we can transform (13) and (14) into

$$
\begin{aligned}
& d s p(\text { Hyp })=\frac{1-\bar{\rho}^{n}}{1+\bar{\rho}^{\delta}-\bar{\rho}^{n}}, \\
& d p s(\text { Hyp })=\frac{1}{1+\bar{\rho}^{\delta}-\bar{\rho}^{n}} .
\end{aligned}
$$

This seems to be a reasonable result, because for $\rho>0$ both $d s p($ Hyp $)$ and $d p s(H y p)$ converge towards 1 for $n \rightarrow \infty$ (and $m$ fixed) and towards 0 for 
$m \rightarrow \infty$ (and $n$ fixed). Note that this is true for any $\rho>0$. Furthermore, the results depend on both $\delta$ and $N$. If $\delta$ is fixed, $\rho>0$, and $N \rightarrow \infty$, then

$$
d s p(H y p)=d p s(H y p)=\frac{1}{1+\bar{\rho}^{\delta}} .
$$

Now look at the case $\gamma=1$ where a prior probability $h=\operatorname{Pr}($ Hyp $)$ is given. This allows the derivation

$$
d s p(H y p)=\operatorname{dps}(H y p)=\frac{h}{h+\bar{h} \bar{\rho}^{\delta}}
$$

from (12). This result converges again towards 1 and 0 for $n \rightarrow \infty$ (and $m$ fixed) and $m \rightarrow \infty$ (and $n$ fixed), respectively. Note that (18) is only a function of $h, \rho$, and $\delta$, but not of $N$.

\subsection{The Model (CL)}

Consider the (L) model that distinguishes between truth telling and lying. As a consequence $z_{1}=z_{2}=0$, we get $d s p(H y p)=d p s(H y p)$ independently of $\gamma$. If prior knowledge is not available, i.e. if $\gamma=0$, it follows from (13) and (14) that

$$
d s p(H y p)=\operatorname{dps}(H y p)=\frac{1}{1+(\bar{r} / r)^{\delta}}
$$

For $m=0$, this result includes Laplace's formula (3) as a special case. Note that $r=\frac{1}{2}$ implies $d s p(H y p)=d p s(H y p)=\frac{1}{2}$. Otherwise, if there are infinitely many positive reports, i.e. for $n \rightarrow \infty$ (and $m$ fixed), we get

$$
d s p(H y p)=d p s(H y p)=\left\{\begin{array}{l}
1, \text { if } r>\frac{1}{2} \\
0, \text { if } r<\frac{1}{2}
\end{array}\right.
$$

On the other hand, if there are infinitely many negative reports, i.e. for $m \rightarrow \infty$ (and $n$ fixed), we obtain

$$
d s p(\text { Hyp })=\operatorname{dps}(\text { Hyp })=\left\{\begin{array}{l}
0, \text { if } r>\frac{1}{2} \\
1, \text { if } r<\frac{1}{2}
\end{array}\right.
$$


Note that (20) and (21) is essentially the Condorcet Jury Theorem discussed in social choice theory [4-7]. The problem with (19), (20), and (21) is that the results only depend on $r$ and $\delta$, but not on $N$ (see [7] for a profound discussion on this).

Now consider the case of $\gamma=1$ with a given prior probability $h=\operatorname{Pr}($ Hyp $)$. This allows the transformation of (12) into

$$
d s p(H y p)=d p s(H y p)=\frac{h}{h+\bar{h}(\bar{r} / r)^{\delta}} .
$$

This result is similar to the one given in (19) and demonstrates the impact of the prior probability $h$. The formula corresponds to the one given by Boole [8]. The parameters on which (22) depends are $h, r$, and $\delta$, but not $N$.

\subsection{The Model (CD)}

Recall that (D) is a generalization of (L). The idea of distinguishing between between two parameters $p$ and $q$ is the same as in the first introductory model in Section 1. If there is no prior knowledge, i.e. if $\gamma=0$, we can transform (13) and (14) into

$$
\begin{aligned}
& d s p(\text { Hyp })=\frac{1-q^{n} \bar{q}^{m}}{1+(p / q)^{n}(\bar{p} / \bar{q})^{m}-q^{n} \bar{q}^{m}}, \\
& d p s(H y p)=\frac{1}{1+(p / q)^{n}(\bar{p} / \bar{q})^{m}-q^{n} \bar{q}^{m}} .
\end{aligned}
$$

Consider the general case in which both $p$ and $q$ are strictly between 0 and 1 . First, consider the special case of $p=q$. This allows to write (23) as

$$
d s p(H y p)=\frac{1-q^{n} \bar{q}^{m}}{2-q^{n} \bar{q}^{m}}=1-d p s(H y p)
$$

So both $d s p($ Hyp $)$ and $d p s($ Hyp $)$ convere towards $\frac{1}{2}$ for both $n \rightarrow \infty$ (and $m$ fixed) or $m \rightarrow \infty$ (and $m$ fixed). This means that an information source of this kind is valueless.

Second, let $p$ be different from $q$. Then $n \rightarrow \infty$ and a fixed $m$ implies 


$$
d s p(\text { Hyp })=\operatorname{dps}(\text { Hyp })=\left\{\begin{array}{l}
1, \text { if } p>q \\
0, \text { if } p<q
\end{array}\right.
$$

Similarly, the case of $m \rightarrow \infty$ and a fixed $n$ implies

$$
d s p(\text { Hyp })=\operatorname{dps}(\text { Hyp })=\left\{\begin{array}{l}
0, \text { if } p>q \\
1, \text { if } p<q
\end{array}\right.
$$

Thus, if the (PI) model is used to describe medical tests, for example, the parameter $p$ is expected to exceed $q$.

Finally, consider the case of $\gamma=1$ and $h=\operatorname{Pr}(H y p)$. By inserting the corresponding parameters into (12), we obtain

$$
d s p(H y p)=\operatorname{dps}(\text { Hyp })=\frac{h}{h+\bar{h}(q / p)^{n}(\bar{q} / \bar{p})^{m}} .
$$

This result reproduces (1) that we obtained in Section 1 with the aid of Bayesian networks (see [2] for details). We get $d \operatorname{sp}($ Hyp $)=d p s(H y p)=h$ for $p=q$, which again means that the information is of no value. If $p$ is different from $q$ and $0<h<1$, the limits for $n \rightarrow \infty$ and $m \rightarrow \infty$ are the same as in (26) and (27).

\subsection{The Model (CPR)}

The next model we analyze here is the one that corresponds to the second introductory example in Section 1. Since $\rho=0$ leads to (CR) and $\rho=1$ to (CP), we suppose $0<\rho<1$ throughout the discussion. We start again with the case of no prior knowledge represented by $\gamma=0$. This allows us to write (13) and (14) as

$$
\begin{aligned}
& d s p(\text { Hyp })=\frac{1-\left(\frac{\bar{\rho} r}{1-\bar{\rho} \bar{r}}\right)^{n}}{1+\left(\frac{\bar{\rho} r}{1-\bar{\rho} \bar{r}}\right)^{n}\left[\left(\frac{1-\bar{\rho} r}{\bar{\rho} \bar{r}}\right)^{m}-1\right]}, \\
& d p s(\text { Hyp })=\frac{1}{1+\left(\frac{\bar{\rho} r}{1-\bar{\rho} \bar{r}}\right)^{n}\left[\left(\frac{1-\bar{\rho} r}{\bar{\rho} \bar{r}}\right)^{m}-1\right]},
\end{aligned}
$$


respectively. Furthermore, we obtain $d s p(H y p)=d p s($ Hyp $)=1$ for $n \rightarrow \infty$ and $d s p($ Hyp $)=d p s($ Hyp $)=0$ for $m \rightarrow \infty$. Finally, if $\delta$ is fixed and $N \rightarrow \infty$, then the result converges to

$$
d s p(\text { Hyp })=d p s(\text { Hyp })=\left\{\begin{aligned}
1, & \text { if } r>\frac{1}{2}, \\
\frac{1}{1+\left(\frac{1-\rho}{1+\rho}\right)^{\delta}}, & \text { if } r=\frac{1}{2}, \\
0, & \text { if } r<\frac{1}{2} .
\end{aligned}\right.
$$

Now consider the case of $\gamma=1$ and $h=\operatorname{Pr}(H y p)$. We can then derive

$$
d s p(H y p)=d p s(H y p)=\frac{h}{h+\bar{h}\left(\frac{\bar{\rho} r}{1-\bar{\rho} \bar{r}}\right)^{n}\left(\frac{1-\bar{\rho} r}{\bar{\rho} \bar{r}}\right)^{m}}
$$

from (12). This formula corresponds to the one obtained in Section 1 with the aid of a Bayesian network. Provided that $0<h<1$, as one would expect, both $d s p(H y p)$ and $d p s(H y p)$ converge towards 1 for $n \rightarrow \infty$ (and $m$ fixed) and towards 0 for $m \rightarrow \infty$ (and $n$ fixed). If $\delta$ is fixed, then $N \rightarrow \infty$ lets (32) converge to

$$
d s p(\text { Hyp })=d p s(\text { Hyp })=\left\{\begin{aligned}
1, & \text { if } r>\frac{1}{2} \\
\frac{h}{h+\bar{h}\left(\frac{1-\rho}{1+\rho}\right)^{\delta}}, & \text { if } r=\frac{1}{2} \\
0, & \text { if } r<\frac{1}{2}
\end{aligned}\right.
$$

Note that this result depends on $\rho$ and $\delta$ only if $r=\frac{1}{2}$.

\subsection{The Model (CYR)}

To conclude this section, let us take a closer look at the (CYR) model. It has an interesting property, namely that a single negative report is enough to set both $d s p($ Hyp $)$ and $d p s($ Hyp $)$ to 0 . For $\gamma=0$, that is if no prior information is available, the general result for $n$ positive and $m$ negative reports is as follows: ${ }^{5}$

\footnotetext{
5 To derive (34) and (35) from (13) and (14), respectively, we assume $0^{0}=1$ to be true. Note that this is not a fundamental mathematical truth, but a definition accepted by most mathematicians and often used to simplify formulas [23].
} 


$$
\begin{aligned}
& d s p(\text { Hyp })=\left\{\begin{array}{r}
1-r^{n}, \text { if } m=0 \\
0, \text { if } m \geq 1
\end{array}\right. \\
& \operatorname{dps}(\text { Hyp })=\left\{\begin{array}{l}
1, \text { if } m=0, \\
0, \text { if } m \geq 1,
\end{array}\right.
\end{aligned}
$$

As long as there are only positive reports, that is for $m=0$ and thus $N=n$, the above result for $d s p(H y p)$ corresponds to formula (4) of the last introductory example. It describes thus the output reliability delivered by a probabilistic algorithm after $N$ successful runs. For $r>0$ and $N \rightarrow \infty$, it converges towards 1 .

In the case of $\gamma=1$ with a given prior distribution $h=\operatorname{Pr}(H y p)$, we can simplify (12) into

$$
d s p(H y p)=d p s(H y p)=\left\{\begin{array}{c}
\frac{h}{h+\bar{h} r^{n}}, \text { if } m=0 \\
0, \text { if } m \geq 1 .
\end{array}\right.
$$

Again, as long as there are only positive reports, this result converges towards 1. On the other hand, a single negative report is sufficient to set both $d s p($ Hyp $)$ and $\operatorname{dps}(H y p)$ to 0.

\section{Conclusion}

This paper approaches the problem of independent and partially reliable information sources from a very general perspective by using the theory of probabilistic argumentation as modeling framework and Dempster-Shafer's theory of evidence as the underlying mathematical mechanisms. The result is a generic model with a number of possible instantiations. The paper illuminates the relationship between the various instantiations and analyses corresponding conclusions. It also discusses the role of prior knowledge and proposes a model in which the influence of a given prior probability is controlled by a continuous parameter $\gamma$.

There are a number of open questions. One of them concerns the problem of choosing the "right" model. We do not exclude the possibility of arguing in favor or against certain models, but we can't and we don't want to give a definite answer here. In our view, the choice of the model crucially depends on the specific application at hand and the nature of the available information. 
In this sense, we think that all models are legitimate. We plan to study some applications in future work.

Another open question is the treatment of dependencies. Relaxing the assumption of independent sources would certainly make the analysis more complicated, but it would also allow us to model a broader class of cases. This important topic is also postponed to future publications.

\section{Acknowledgements}

Special thanks to: (1) the anonymous reviewers for their insightful, detailed, and very constructive comments; (2) Michael Wachter for proof-reading and helpful discussions.

\section{References}

[1] R. Haenni, S. Hartmann, A general model for partially reliable information sources, in: P. Svensson, J. Schubert (Eds.), FUSION'04, 7th International Conference on Information Fusion, Vol. I, Stockholm, Sweden, 2004, pp. 153160 .

[2] L. Bovens, S. Hartmann, Bayesian Epistemology, Oxford University Press, 2003.

[3] P. S. Laplace, Théorie Analytique des Probabilités, 3rd Edition, Courcier, Paris, 1820.

[4] Marquis de Condorcet, Essai sur l'application de l'analyse à la probabilité des décisions rendues à la pluralité des voix, L'Imprimerie Royale, Paris, France, 1785 .

[5] D. Black, Theory of Committees and Elections, Cambridge University Press, Cambridge, 1958.

[6] C. List, R. E. Goodin, Epistemic democracy: Generalizing the Condorcet jury theorem, Journal of Political Philosophy 9 (3) (2001) 277-306.

[7] C. List, On the significance of the absolute margin, British Journal for the Philosophy of Science 55 (3) (2004) 521-544.

[8] G. Boole, The Laws of Thought, Walton and Maberley, London, 1854.

[9] R. Motwani, P. Raghavan, Randomized Algorithms, Cambridge University Press, 1995.

[10] M. O. Rabin, Probabilistic algorithm for primality testing, Journal of Number Theory 12 (1980) 128-138. 
[11] A. Dempster, Upper and lower probabilities induced by a multivalued mapping, Annals of Mathematical Statistics 38 (1967) 325-339.

[12] G. Shafer, The Mathematical Theory of Evidence, Princeton University Press, 1976.

[13] P. Smets, R. Kennes, The transferable belief model, Artificial Intelligence 66 (1994) 191-234.

[14] J. Kohlas, P. A. Monney, A Mathematical Theory of Hints. An Approach to the Dempster-Shafer Theory of Evidence, Vol. 425 of Lecture Notes in Economics and Mathematical Systems, Springer-Verlag, 1995.

[15] R. Haenni, J. Kohlas, N. Lehmann, Probabilistic argumentation systems, in: J. Kohlas, S. Moral (Eds.), Handbook of Defeasible Reasoning and Uncertainty Management Systems, Volume 5: Algorithms for Uncertainty and Defeasible Reasoning, Kluwer Academic Publishers, 2000, pp. 221-288.

[16] R. Haenni, N. Lehmann, Probabilistic argumentation systems: a new perspective on Dempster-Shafer theory, International Journal of Intelligent Systems (Special Issue: the Dempster-Shafer Theory of Evidence) 18 (1) (2003) 93-106.

[17] R. Haenni, Unifying logical and probabilistic reasoning, in: ECSQARU'05, 8th European Conference on Symbolic and Quantitative Approaches to Reasoning under Uncertainty, Barcelona, Spain, 2005 (accepted for publication).

[18] J. Kohlas, Information Algebras: Generic Stuctures for Inference, SpringerVerlag, London, 2003.

[19] R. Haenni, Ignoring ignorance is ignorant, Tech. rep., Center for Junior Research Fellows, University of Konstanz (2003).

[20] J. H. Lambert, Neues Organon oder Gedanken über die Erforschung und Bezeichnung des Wahren und dessen Unterscheidung vom Irrtum und Schein, Johann Wendler, Leipzig, 1764.

[21] J. Kohlas, D. Berzati, R. Haenni, Probabilistic argumentation systems and abduction, Annals of Mathematics and Artificial Intelligence 34 (1-3) (2002) $177-195$.

[22] P. Walley, Statistical Reasoning with Imprecise Probabilities, Monographs on Statistics and Applied Probability 42, Chapman and Hall, London, UK, 1991.

[23] D. E. Knuth, Two notes on notation, American Mathematical Monthly 99 (5) (1992) 403-422. 